\section{Preliminary investigation on the use of allyl isothio- cyanate to increase the shelf-life of gilthead sea bream (Sparus aurata) fillets}

\author{
Filippo Giarratana, Chiara Crinò, \\ Daniele Muscolino, Chiara Beninati, \\ Graziella Ziino, Alessandro Giuffrida,
} Antonio Panebianco

Department of Veterinary Sciences, University of Messina, Italy

\begin{abstract}
The aim of this work is to evaluate the activity of allyl isothiocyanate (AITC) against fish spoilage bacteria (specific spoilage organisms; SSOs) as well as its possible use in gilthead sea bream (Sparus aurata) fillets to extend their shelf-life. In this regard, in vitro tests are carried out in order to evaluate the inhibitory activity of AITC and its vapours on several strains of SSOs. The AITC effect on the shelflife of sea bream fillets was made by putting them in plastic trays hermetically closed with the addition AITC. Microbiological and sensorial evaluations were made on fish fillets during storage. Treated fillets maintained microbial populations at a significantly lower level compared with the control samples during storage, showing better sensorial characteristics. Therefore, the use of AITC's vapours seems to be a new and interesting alternative way to increase fish product shelf-life.
\end{abstract}

\section{Introduction}

Allyl isothiocyanate (AITC) is a natural compound from plants belonging to the family Cruciferae. It is found in the seeds, stem, leaves, and roots of cruciferous plants including horseradish, black and brown mustard, cabbage, Brussels sprouts, broccoli, cauliflower, kohlrabi, kale, turnip, rutabaga, watercress, wasabi, radish and papaya (EFSA, 2010). It appears as an oily liquid, insoluble in water, with molecular weight of $99.1 \mathrm{~g} / \mathrm{mol}$. Commercially it is produced by the reaction of chloride of allyl and thiocyanate of potassium, or by dry distillation of seeds. As known, AITC is bitter in taste with a strong, pungent and mustard-like smell and it is authorized as a flavouring substance in foods by the European Regulation EC No 872/2012 (European Commission, 2012). Moreover the AITC possesses also antimicrobial, antifungal, insecti- cide and nematocidal properties (Minarini et al., 2014; Giarratana et al.., 2015). The antimicrobial activity of AITC was demonstrated on several microorganisms, especially foodborne pathogens, including Escherichia coli 0157:H7, Salmonella montevideo, Salmonella typhimurium and Listeria monocytogenes (Delaquis and Sholberg, 1997; Lin et al., 2000a, 2000b; Luciano and Holley, 2009). The antibacterial effectiveness of AITC against foodborne pathogens was also demonstrated in several food matrix such as meat sauce (Chacon et al., 2006b), minced beef (Chacon et al., 2006a), fresh chicken breasts (Shin et al., 2010), and cheese (Winther and Nielsen, 2006). Although its antibacterial activity is well known on foodborne agent, few studies have been carried out on its effectiveness against spoilage bacteria of fishery products (Pang et al., 2013). Microorganisms, notably SSOs (specific spoilage organisms) are in fact the major cause of spoilage in seafood products. Therefore, the aim of this work is to assess the antibacterial activity of AITC on the growth of SSOs.

\section{Materials and Methods}

\section{In vitro test}

A total of 39 fish isolates belonging to the group of SSOs were used for the assessment of AITC vapour inhibiting activity in vitro. Bacteria strains were isolated from skin and muscle of farmed gilthead sea bream (Sparus aurata). Strains were: 2 Pseudomonas fluorescens/putida; 1 Pseudomonas fluorescens; 2 Pseudomonas stutzeti; 6 Pseudomonas putida; 2 Pseudomonas syringae; 1 Pseudomonas gessardifluorescens; 1 Pseudomonas mandorli; 3 Pseudomonas fragi; 3 Pseudomonas putrefaciens; 11 Shewanella algae/putrefaciens; 1 Shewanella putida; 4 Shewanella putrefaciens; 1 Shewanella baltica; 1 Achromobacter spp.

Agar gel diffusion technique was used in order to assess the effectiveness of AITC against SSOs. Three different concentrations of the compound $(2,4$ and $8 \mu \mathrm{L})$ were tested. Muller Hinton Agar plates (MHA) (Oxoid Ltd., Basinkstoke, Hampshire, England) were inoculated with each strain suspension and sterile discs (Biolife, Milan, Italy) soaked with AITC were placed on the surface of MHA. Plates were then incubated at $30^{\circ} \mathrm{C}$ for $24 \mathrm{~h}$. Furthermore, a test in tight closed jar was carried out in order to evaluate the inhibiting activity of AITC vapours. Four MHA plates inoculated with strain suspensions were placed into tight closed jar with a glass becker containing a filter paper soaked with different amounts of AITC $(1,300$ , 100 and $30 \mu \mathrm{L}$ ). The paper filters worked as an AITC carrier. Control treatments consisted in jar with filter papers added with sterile-distilled
Correspondence: Filippo Giarratana, Department of Veterinary Sciences, University of Messina, Viale Annunziata, 98168 Messina, Italy Tel: +39.090 .3503768 .

E-mail: fgiarratana@unime.it

Key words: Allyl isothiocyanate; Specific spoilage organisms; Gilthead sea bream fillets; Shelf-life.

Acknowledgments: this research was supported by the Research project PON02_3362185 "innoVaqua".

Received for publication: 8 July 2014.

Revision received: 27 January 2015.

Accepted for publication: 21 January 2015

This work is licensed under a Creative Commons Attribution 3.0 License (by-nc 3.0).

(C) Copyright F. Giarratana et al., 2015

Licensee PAGEPress, Italy

Italian Journal of Food Safety 2015; $4: 4512$

doi:10.4081/ijfs.2015.4512

water. The jars were sealed immediately and were incubated at $30^{\circ} \mathrm{C}$ for $24 \mathrm{~h}$. The inhibitory effect of AITC against SSOs strains tested was determined by evaluation of bacterial growth after incubation.

\section{Test on sea bream fillets}

The present study was carried out on 48 fillets of gilthead sea bream (Sparus aurata) from a sea-based aquaculture facility located in Sicily (Italy) stored at $6^{\circ} \mathrm{C}$ with and without addition of AITC. Three groups were created: i) group 1: control; ii) group 2: fillets permanently exposed to AITC vapours (10 $\mu \mathrm{L}$ AITC); iii) group 3: fillets exposed to AITC vapours $(10 \mu \mathrm{L}$ AITC) only for one hour. Each fillet was packed in transparent polypropylene trays hermetically sealed and stored at $6^{\circ} \mathrm{C}$ for six days. The addition of AITC was made with a filter paper (2.5 $\times 2.5 \mathrm{~cm})$ soaked with the compound. The paper filters worked as an AITC vapours carrier. Fillets were stored at $6^{\circ} \mathrm{C}$ in order to accelerate the spoilage of products and to evaluate the effectiveness of AITC vapours against spoilage bacteria under suboptimal temperature conditions. Microbiological and sensorial evaluations were made at $0,24,48,72,96,120$ and 144 hours of storage. Count of SSOs was made on Lyngby Iron Agar (Oxoid Ltd) incubated at $25^{\circ} \mathrm{C}$ for $3-5$ days. Sensorial evaluations on raw and cooked fillets were carried out by using the quality index method (QIM) scheme as described in Tables 1 and 2. Fish fillets were cooked in a microwave for $1 \mathrm{~min}(300 \mathrm{w})$.

\section{Results and Discussion}

\section{In vitro test}

In vitro test revealed a strong activity of AITC against all strains tested. No bacterial 
growth was observed at all concentrations used.

\section{Test on sea bream fillets}

Figure 1 shows the results of microbiological evaluation. The initial SSOs of sea bream fillets was $3.38 \pm 0.17 \log \mathrm{CFU} / \mathrm{g}$. Treated samples with AITC maintained microbial populations at a significantly lower level compared with the control samples during the storage. After the 144 hours of storage, SSOs count reached $5.68 \pm 0.03 \log \mathrm{CFU} / \mathrm{g}$ and $6.58 \mathrm{log}$ $\mathrm{CFU} / \mathrm{g}$ in group 2 and 3 respectively. Control group reached the same value just after 72 hours of storage and at 144 hours had bacterial load of $9.28 \pm 0.03 \log$ CFU/g.

Figures 2 and 3 show the results of sensorial evaluation. During the first days of storage every groups presented the same sensorial characteristics. Treated fillets (groups 2 and 3 ) showed a marked AITC odour after opening the

Table 1. Quality index method scheme for raw fillets.

\begin{tabular}{ll} 
Odour & \\
$\quad$ Fresh, salt, of seaweed & 0 \\
Neutral & 1 \\
$\quad$ Stale & 2 \\
Colour & \\
$\quad$ Whitish, lucid & 0 \\
$\quad$ Grayish & 1 \\
$\quad$ Yellowish, matt & 2 \\
Texture & \\
$\quad$ Hard-elastic & 0 \\
$\quad$ Elastic & 1 \\
$\quad$ Flaccid and soft & 2 \\
Isothiocyanate's smell & \\
$\quad$ Absent & 0 \\
$\quad$ Barely detectable & 1 \\
$\quad$ Moderate & 2 \\
Intense & 3 \\
\hline
\end{tabular}

Table 2. Quality index method scheme for cooked fillets.

\begin{tabular}{ll}
\hline Odour & \\
Typical & 0 \\
Neutral & 1 \\
Acrid & 2 \\
Taste & \\
Typical & 0 \\
Neutral & 1 \\
$\quad$ Bitter & 2 \\
\hline Isothiocyanate's smell & \\
$\quad$ Absent & 0 \\
$\quad$ Moderate garlicky & 1 \\
$\quad$ Garlicky & 2 \\
Isothiocyanate's taste & \\
Absent & 0 \\
Moderate garlicky & 1 \\
Garlicky & 2 \\
\hline
\end{tabular}

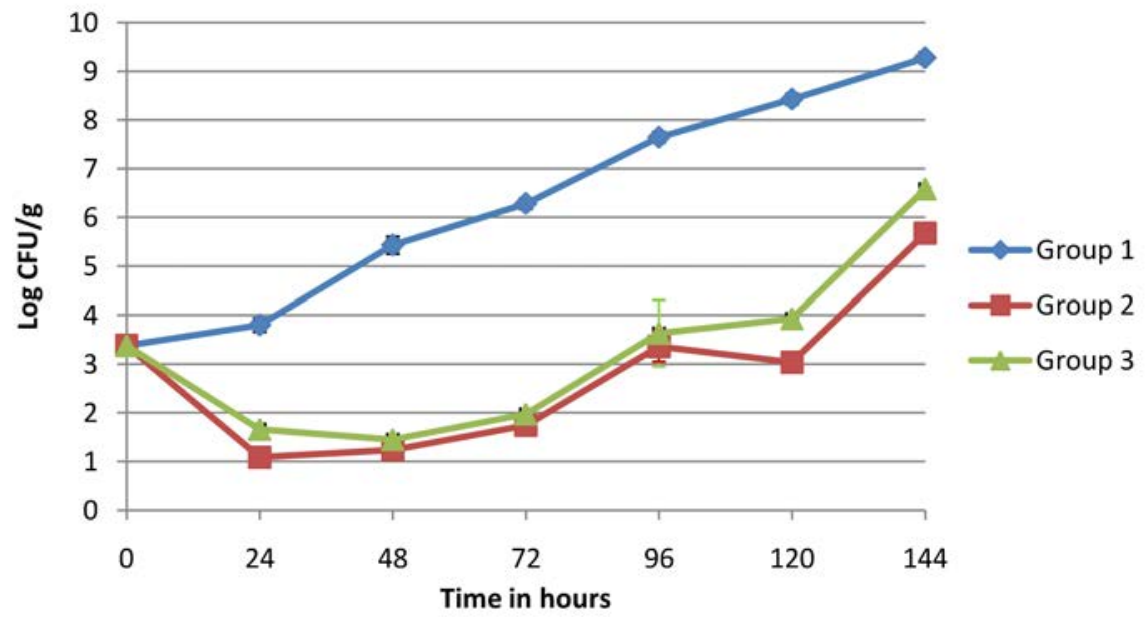

Figure 1. Trend of specific spoilage organisms in raw fillets. Group 1, control; Group 2, fillets permanently exposed to allyl isothiocyanate; Group 3, fillets exposed to allyl isothiocyanate for one hour.

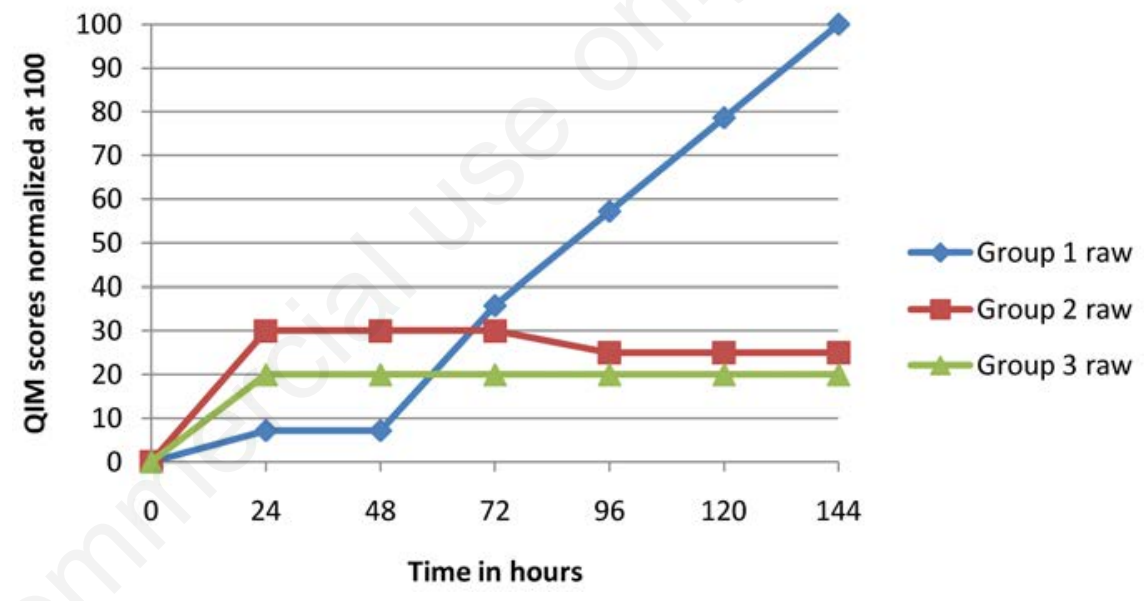

Figure 2. Quality index method scores for raw fillets. Group 1, control; Group 2, fillets permanently exposed to allyl isothiocyanate; Group 3, fillets exposed to allyl isothiocyanate for one hour.

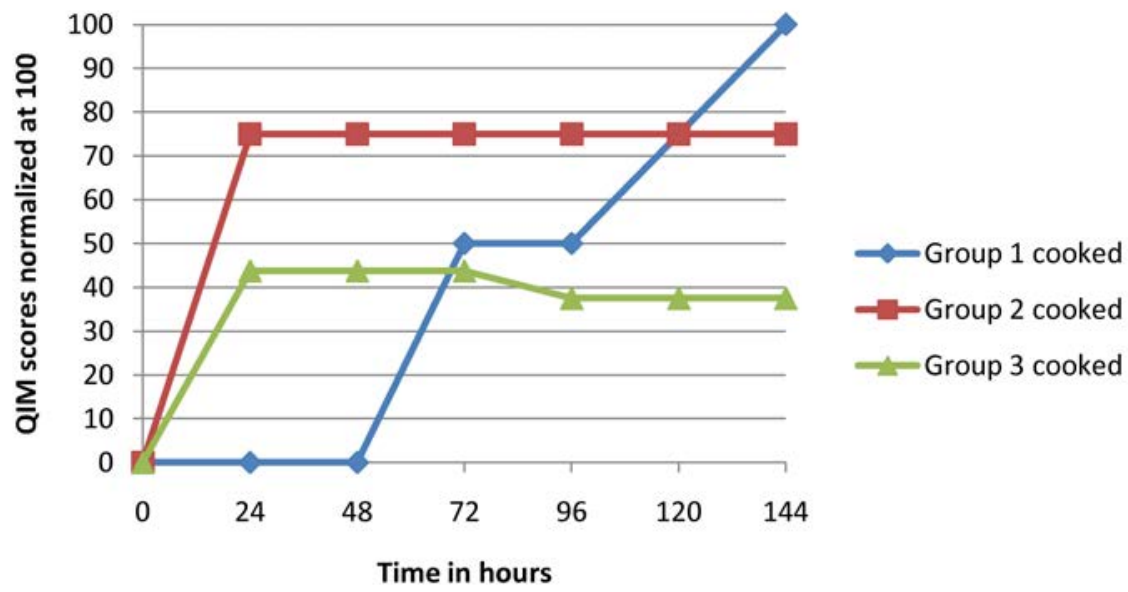

Figure 3. Quality index method scores for cooked fillets. Group 1, control; Group 2, fillets permanently exposed to allyl isothiocyanate; Group 3, fillets exposed to allyl isothiocyanate for one hour. 
package, which disappeared after air exposure in few seconds. After 96 hours of storage, control fillets had a neutral or slight acrid flavour, while the texture tended to be flaccid. On the contrary, treated fillets (groups 2 and 3) maintained constant and fully acceptable features until the last day. After cooking, treated fillets showed a slight garlic odour, stronger when fillets was still hot, and perceptible even to chew, but not nasty. At the end of storage (144 hours), treated fillets with AITC were preferred by panellist because of their desirable aspect and flavour.

\section{Conclusions}

Results show a high effectiveness of AITC against SSOs in vitro and in raw sea bream fillets stored at refrigerate conditions. QIM results show that AITC addition does not affect sensorial characteristics with the exception of a garlic odour of cooked product that, however, did not appear unpleasant to panellists. The use of AITC's vapours on fishery products could be a good preservative technique in order to extend the shelf-life of raw fish fillets. This study showed that AITC was an effective antimicrobial agent to inhibit the growth of SSOs, because it provided 2 times longer shelf life of fresh sea bream fillets than the controls. The continuous exposure to AITC of fillets showed more effective antimicrobial effect on SSOs. In addition, treated fillets maintained desirable sensorial characteristics during storage. However, further investigations need to be conducted in order to evaluate the antimi- crobial effectiveness of AITC's vapours at lower concentrations, and to optimize the range of AITC concentration acceptable by consumers.

\section{References}

Chacon PA, Buffo RA, Holly RA, 2006a. Inhibitory effects of microencapsulated allyl isothiocyanate (AIT) against Escherichia coli 0157:H7 in refrigerated, nitrogen packed, finely chopped beef. Int J Food Microbiol 107:231-7.

Chacon PA, Muthukumarasamy K, Holley RA, 2006b. Elimination of Escherichia coli 0157:H7 from fermented dry sausages at an organoleptically acceptable level of microencapsulated allyl isothiocyanate. Appl Environ Microb 75:3096-102.

Delaquis PJ, Sholberg PL, 1997. Antimicrobial activity of gaseous allyl isothiocyanate. $\mathrm{J}$ Food Protect 8:943-7.

EFSA, 2010. Scientific opinion on the safety of allyl isothiocyanate for the proposed use as a food additive. EFSA J 8:1943.

European Commission, 2012. Commission implementing regulation No of 1 October 2012 adopting the list of flavouring substances provided for by Regulation (EC) No 2232/96 of the European Parliament and of the Council, introducing it in Annex I to Regulation (EC) No 1334/2008 of the European Parliament and of the Council and repealing Commission Regulation (EC) No 1565/2000 and Commission Decision 1999/217/EC, 872/2012/EU. In: Official Journal, L 267/1, 02.10.2012.
Giarratana F, Panebianco F, Muscolino D, Beninati C, Ziino G, Giuffrida A, 2015. Effect of allyl isothiocyanate against Anisakis larvae during the anchovy marinating process. J Food Protect 78:767-71.

Lin CM, Kim J, Du WX, Wei CI, 2000a. Bactericidal activity of Isothiocyanate against pathogens on fresh produce. $\mathrm{J}$ Food Protect 63:25-30.

Lin CM, Preston JF, Wei CI, 2000b. Antibacterial mechanism of allyl isothiocyanate. J Food Protect 63:727-34.

Luciano FB, Holley RA, 2009. Enzymatic inhibition by allyl isothiocyanate and factors affecting its antimicrobial action against Escherichia coli 0157H7. Int J Food Microbiol 131:240-5.

Minarini A, Milelli A, Fimognari C, Simoni E, Turrini E, Tumiatti V, 2014. Exploring the effects of isothiocyanates on chemotherapeutic drugs. Expert Opin Drug Met 10:2538 .

Pang YH, Sheen S, Zhou S, Liu L, Yam KL, 2013. antimicrobial effects of allyl isothiocyanate and modified atmosphere on pseduomonas aeruginosa in fresh catfish fillet under abuse temperatures. J Food Sci 78:555-9.

Shin J, Harte B, Ryser E, Selke S, 2010. Active packaging of fresh chicken breast, with allyl isothiocyanate (AITC) in combination with modified atmosphere packaging (MAP) to control the growth of pathogens. J Food Sci 75:65-71.

Winther M, Nielsen PV, 2006. Active packaging of cheese with allyl isothiocyanate, an alternative to modified atmosphere packaging. J Food Protect 69:2430-5. 